\title{
Demographic Population Cycles in Infectious Salmon Anemia Models
}

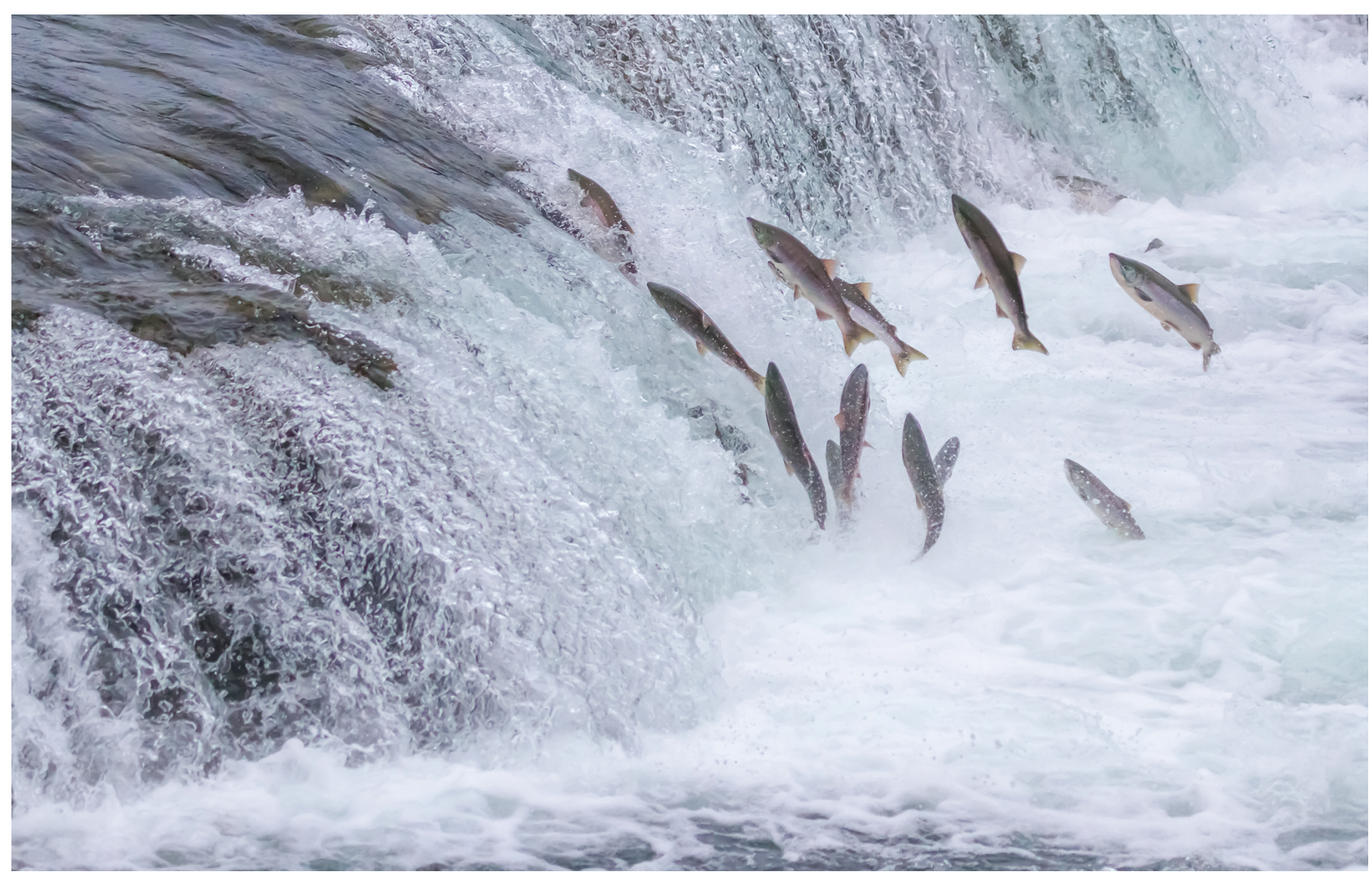

\section{Abdul-Aziz Yakubu}

\section{Introduction}

Salmon, for example Atlantic and Pacific salmon, is the common name of several species of the Salmonidae family of fish. Trout, for example brown and seawater trout, is the name of some of the others in the family. Salmon are typically anadromous. That is, they are born in fresh water, migrate to the ocean, then return to fresh water to reproduce.

Abdul-Aziz Yakubu is a professor in the Department of Mathematics at Howard University. His email address is ayakubu@howard. edu.

Communicated by Notices Associate Editor Reza Malek-Madani.

For permission to reprint this article, please contact:

reprint-permission@ams.org.

DOI: https://doi.org/10.1090/noti2015
Several of the salmon species are available from both wild and farmed sources. In the past forty years, the farmed salmon industry has grown substantially. Today, approximately 60 percent of the world's salmon production is farmed. In 2015, more than 2,200,000 tons of farmed salmon were produced, while in comparison around 880,000 tons of wild salmon were caught [7], [13]. The majority of salmon farms consist of a collection of net cages, known as "open net-cages," that are simply suspended in the water with no barrier between the farm and the surrounding environment. Typically, the open net-cages are located in areas along the coast where ocean currents deliver oxygen to the farmed salmon while their 
wastes (feces and feed) disperse to the environment [12], [17], [18]. Due to salmon's high content of protein and omega-3 fatty acids, its consumption reduces the risk of cardiovascular diseases. Also, salmon is a good source of minerals and vitamins. The European Union, the United States, and China make up over 70 percent of the global market for Atlantic salmon, and in each of these places consumption of both farmed and wild salmon is increasing [7].

Infectious salmon anemia virus (ISAv) is the cause of infectious salmon anemia (ISA), a serious viral fish disease that affects mostly farmed Atlantic salmon (Salmo salar) in several areas of the world. This highly contagious disease can be insidious, with an initially low mortality rate. However, the cumulative ISA mortality rate can sometimes exceed 90 percent if it remains unchecked. ISA has caused significant mortality with severe implications for production economics among salmon farms in Northern Europe, Canada, Maine, and Chile [6], [8]. In 2000, ISA devastated the salmon industry of the Faroe Islands, and in 1998-99 an epizootic in Scotland cost an estimated $\$ 32,000,000$ to eliminate, and millions of fish were culled in ISA control efforts. ISA is a major threat not only to the viability of farmed Atlantic salmon but also to dwindling stocks of wild Atlantic salmon. Production losses, loss of export markets, and the associated social impacts make elimination and control of the ISA $v$ infection a priority for the Atlantic salmon industry. Currently, there is no treatment for fish infected with ISAv. Furthermore, our current understanding of the epidemiology of ISA is still incomplete, and this complicates its control. ISA-infected salmon pose no human health threat.

Mathematical models of infectious disease transmission dynamics are ubiquitous in the literature. These models can play important roles in helping to quantify possible infectious disease control and mitigation strategies. The models allow us to first test a variety of control and mitigation strategies via computer simulations before actual implementation on real populations. An important epidemiological threshold parameter that quantifies disease invasion or extinction in a population is the basic reproduction number or basic reproduction ratio, denoted by $\mathcal{R}_{0}[1]$ and [2]. $\mathcal{R}_{0}$ is defined as the average number of secondary cases produced by a single infectious individual introduced into a totally susceptible population. Consequently, values of $\mathcal{R}_{0}<1$ imply that the number of infections will decrease and the disease eventually dies out as the chain of transmission cannot be maintained. However, values of $\mathcal{R}_{0}>1 \mathrm{im}$ ply that the number of infections will increase, the disease invades, and an epidemic occurs in the population. For example, it was estimated that $\mathcal{R}_{0}$ of measles is between 12 and 18. That is, each individual infected with measles would, on average, transmit the measles infection to 12-18 other individuals in a totally susceptible population. To help quantify possible ISA disease control and mitigation strategies, in this paper, we introduce discrete-time mathematical models of ISA disease transmission dynamics in salmon fish farms and wild fisheries in close proximity. For these models, we compute $\mathcal{R}_{0}$, the threshold parameter for ISA disease elimination or invasion.

Epidemiological studies suggest that horizontal transmission of ISAv occurs readily within salmon open netcages. The infection also takes place, although more slowly, between salmon in different nets at a site, as well as between farms. ISA $v$ probably infects fish through the gills, but ingestion has not been ruled out. Unlike farmed salmon, wild salmon live and breed in their native bodies of water, and humans have no control over their breeding, feeding, or health. Wild salmon are known to swim over long distances with no restriction. The locations of salmon farms among wild salmon migratory routes are known to raise risk of ISA infections in nearby wild salmon populations [9], [10], [14]. Determining the possible role wild salmon play in ISA $v$ transfer to nearby salmon farms is an interesting question.

In a 2016 study of ISA disease transmission dynamics, Milliken and Pilyugin used a deterministic two-patch diffusion linked continuous-time system of ordinary differential equations with the logistic (noncyclic) salmon growth function to model ISA $v$ infections in farmed salmon populations that are in close proximity to a wild salmon migratory route [12]. The ODE model of Milliken and Pilyugin assumed that, in the absence of the ISA infection, the salmon population is at rest on a static equilibrium point. However, salmon populations are known to exhibit density-dependent population cycles [15], [18]. A striking example of oscillations in salmon populations is the 4year cycle in the number of spawning sockeye salmon (Oncorhynchus nerka) that return to their native streams. Also, pink salmon (Oncorhynchus gorbuscha) exhibits enormous variations in existence and amplitude of 2-year cycles in abundance throughout its range in the Pacific Ocean. Simple single species nonlinear discrete-time population models, such as one hump maps, are capable of generating such period $k$ or $k$-years population cycles and chaotic dynamics, even without environmental stochasticity. The Ricker model, a discrete-time nonlinear population model first used by Ricker in 1954 to study population cycles in fish, is capable of generating density-dependent period $k$ population cycles in a demographic equation (in the absence of a disease; see [11] and [15]). In this article, we develop a discrete-time six-dimensional viral dispersallinked farmed-wild salmon ISA model with Ricker demographic dynamics. Others have studied discrete-time infectious disease models; for example see [1] and [2]. As in [12], we consider a farmed-wild salmon system that 
consists of a salmon farm in close proximity to a wild salmon population. We assume that the farmed salmon population, located in open net-cages, and the wild salmon population, located in the nearby open body of water, are not physically in direct contact but are connected by the ISA virus in the water environment via the dispersion or diffusion of the virus between the open net-cages and the nearby open body of water. To capture the salmon demographic population cycles, unlike in [12], we assume that susceptible farmed and wild salmon populations exhibit Ricker growth or recruitment (or birth) functions, and the proportion of ISA $v$ that disperses from the open net-cages to the open body of water is not equal to the proportion of ISA $v$ that disperses from the nearby open water to the cages. Furthermore, the mortality rates in the farmed and wild salmon populations are not identical. We also assume that the ISA $v$-infected salmon populations cannot reproduce, and there are no vertical ISA $v$ transmissions. That is, we assume that all newborn salmon populations are susceptible.

In a recent paper [18], van den Driessche and Yakubu introduced a discrete-time model of ISA without dispersion. Several authors have studied population models that allow for the interaction of density-dependent demographic dynamics and dispersion with or without explicit disease dynamics (for example, see [3]). In this article, our emphasis is on exploring the effects of ISA $v$ dispersion on the invasion or elimination of the ISA disease infection and the basic reproduction number, $\mathcal{R}_{0}$, where the salmon demographic dynamics is periodic. When $\mathcal{R}_{0}<1$ and the demographic equation has a locally asymptotically stable period $k$ population cycle, we prove the local asymptotic stability of the dispersal-linked farmed-wild salmon ISA disease-free period $k$ population cycle, and the disease dies out in the farmed and wild salmon populations. Also, under the same period $k$ demographic assumption, we prove that the ISA disease-free period $k$ population cycle is unstable and the disease invades the farmed and wild salmon populations when $\mathcal{R}_{0}>1$. In addition, we use simulations to explore the relationships between high and low proportion of viral dispersals from the farmed cages to the nearby open body of water on ISA invasion or elimination.

\section{Farmed-Wild Salmon Viral Dispersal Linked ISA Model}

To introduce a farmed-wild salmon discrete-time ISA model with viral dispersion, we assume that at each time $t \in\{0,1,2, \ldots\}$, each live salmon is either susceptible farmed, $S_{t}^{F}$, or wild, $S_{t}^{W}$, or infectious farmed, $I_{t}^{F}$, or wild, $I_{t}^{W}$. That is, we let $S_{t}^{\Lambda}, I_{t}^{\Lambda}$, and $N_{t}^{\Lambda}=S_{t}^{\Lambda}+I_{t}^{\Lambda}$, respectively, denote the population size of susceptible, infectious, and total population of farmed (respectively, wild) salmon at each time $t$ when $\Lambda=F$ (respectively, when $\Lambda=W$ ). Once infected, salmon do not recover from the ISA disease. At each time $t \in\{0,1,2, \ldots\}$, we denote the virus population size in the open net-cages by $V_{t}^{F}$, and the nearby virus population size in the open body of water by $V_{t}^{W}$. Thus, we use an SI farmed-wild ISA epidemic model with virus dispersion and no recovery class to describe the salmon populations.

Per each unit time interval, $d_{F} \in(0,1)$ (respectively, $\left.d_{W} \in(0,1)\right)$ is the fraction of farmed (respectively, wild) salmon that die "naturally," $\widehat{d}_{F}=\left(1-d_{F}\right)$ (respectively, $\left.\widehat{d}_{W}=\left(1-d_{W}\right)\right)$ is the fraction of farmed (respectively, wild) salmon that survives, $\mu_{F} \in(0,1)$ (respectively, $\left.\mu_{W} \in(0,1)\right)$ is the farmed (respectively, wild) constant ISA-induced mortality, $\widehat{\mu}_{F}=\left(1-\mu_{F}\right)$ (respectively, $\widehat{\mu}_{W}=$ $\left.\left(1-\mu_{W}\right)\right)$ is the fraction of ISA infectious farmed (respectively, wild) salmon that survives the infection, $d_{V}^{F} \in(0,1)$ (respectively, $\left.d_{V}^{W} \in(0,1)\right)$ is the constant fraction of virus that is cleared in the open net-cages (respectively, open body of water), and $\widehat{d}_{V}^{F}=\left(1-d_{V}^{F}\right)$ (respectively, $\widehat{d}_{V}^{W}=$ $\left.\left(1-d_{V}^{W}\right)\right)$ is the fraction of virus that survives in the open net-cages (respectively, open body of water). Salmon have high annual mortality rates at sea compared to other marine fisheries. For Atlantic salmon at sea, it is estimated that $65 \% \leq d_{W} \leq 85 \%$. Furthermore, Atlantic salmon at sea have more predators, such as larger pisciverous fish (striped bass, cod, haddock), birds, and mammals. Thus, $d_{F}<d_{W}$. Since ISA is a viral disease of farmed Atlantic salmon, $\mu_{W}<\mu_{F}$ and $d_{V}^{W} \geq d_{V}^{F}$.

In each unit interval, we assume that a fraction of susceptible farmed salmon, $\theta_{F} \in(0,1)$, becomes infected from direct contact with infectious farmed salmon with probability function $\widehat{\varphi}_{F}\left(I_{t}^{F}\right)=\left(1-\varphi_{F}\left(I_{t}^{F}\right)\right)$, and the remaining fraction, $\left(1-\theta_{F}\right) \in(0,1)$, becomes infected via contact with the ISA $v$ in the open net-cages with probability function $\hat{\varphi}_{V}^{F}\left(V_{t}^{F}\right)=\left(1-\varphi_{V}^{F}\left(V_{t}^{F}\right)\right)$ for each time $t \in\{0,1,2, \ldots\}$. Similarly, we assume that a fraction of wild susceptible salmon, $\theta_{W} \in(0,1)$, becomes infected from direct contact with infectious wild salmon with probability function $\widehat{\varphi}_{W}\left(I_{t}^{W}\right)=\left(1-\varphi_{W}\left(I_{t}^{W}\right)\right)$ and the remaining fraction, $\left(1-\theta_{W}\right) \in(0,1)$, becomes infected via contact with the ISA virus in the open body of water with probability function $\widehat{\varphi}_{V}^{W}\left(V_{t}^{W}\right)=\left(1-\varphi_{V}^{W}\left(V_{t}^{W}\right)\right)$, where the "escape" functions

$$
\varphi_{F}, \varphi_{W}, \varphi_{V}^{F}, \varphi_{V}^{W}: \mathbb{R}_{+} \rightarrow[0,1]
$$

are nonlinear decreasing smooth concave up functions with $\varphi_{F}(0)=\varphi_{W}(0)=\varphi_{V}^{F}(0)=\varphi_{V}^{W}(0)=1, \varphi_{F}^{\prime}<0$, $\varphi_{W}^{\prime}<0,\left(\varphi_{V}^{F}\right)^{\prime}<0,\left(\varphi_{V}^{W}\right)^{\prime}<0, \varphi_{F}^{\prime \prime}>0, \varphi_{W}^{\prime \prime}>0,\left(\varphi_{V}^{F}\right)^{\prime \prime}>0$, and $\left(\varphi_{V}^{W}\right)^{\prime \prime}>0$. Thus, in each unit time interval, we assume that the probability of exposure of an individual salmon to multiple ISAv infection pathways is very low and so can be ignored. 
To introduce specific examples of the escape functions, $\varphi_{\Lambda}$ and $\varphi_{V}^{\Lambda}$ for each $\Lambda \in\{F, W\}$, we assume throughout that disease infections are modeled as Poisson processes, and for a single infectious individual fish, we let $\beta_{\Lambda}$ or $\beta_{V}^{\Lambda}$ be the mean number of occurrences of infections in the unit interval for each $\Lambda$. Then

$$
\begin{aligned}
\varphi_{\Lambda}\left(I^{\Lambda}\right)=\exp \left(-\beta_{\Lambda} I^{\Lambda}\right) & \\
& \left(\text { respectively, } \varphi_{V}^{\Lambda}\left(V^{\Lambda}\right)=\exp \left(-\beta_{V}^{\Lambda} V^{\Lambda}\right)\right)
\end{aligned}
$$

equals the fraction of susceptible salmon that escapes from infection after coming in contact with $I^{\Lambda}$ (respectively, $V^{\Lambda}$ ) infectious salmon (respectively, ISA $v$ ) in the unit time interval.

We account for virus shedding by the infectious farmed and wild salmon populations. During each unit time interval, $\delta_{F} I_{t}^{F}$ and $\delta_{W} I_{t}^{W}$ are respectively the population of virus shed by the infectious farmed salmon into the open netcages and nearby wild salmon populations into the open body of water, where $\delta_{F}, \delta_{W}>0$. In each unit time interval, a constant fraction of ISA $v$ in the open net-cages, $m_{F}$ $\in(0,1)$, disperses to the nearby open body of water, while the constant fraction of ISA $v$ in the nearby open body of water, $m_{W} \in(0,1)$, disperses to the open net-cages.

To allow cyclic salmon demographic dynamics, we let

$$
g_{F}, g_{W}: \mathbb{R}_{+} \rightarrow \mathbb{R}_{+}
$$

denote the nonlinear differentiable Ricker recruitment (or birth) function of susceptible farmed (respectively, wild) salmon into the susceptible farmed (respectively, wild) salmon class per unit time interval, where

$$
\begin{aligned}
& g_{F}\left(S_{t}^{F}\right)=r_{F} S_{t}^{F} \exp \left(-b_{F} S_{t}^{F}\right) \\
& \text { and } g_{W}\left(S_{t}^{W}\right)=r_{W} S_{t}^{W} \exp \left(-b_{W} S_{t}^{W}\right) .
\end{aligned}
$$

For the farmed (respectively, wild) salmon, the parameter $r_{F}$ (respectively, $r_{W}$ ) is the density-independent probability of survival from egg to age 1 , and $b_{F}$ (respectively, $b_{W}$ ) is the coefficient of density-dependent mortality [15]. The Ricker model is an example of simple spawner-recruitment and per capita growth function that has played fundamental roles in fishery science for many years.

Our farmed-wild salmon ISA model implicitly assumes three distinct temporal phases. In both the open net-cages and nearby open body of water, at the end of each unit time interval, susceptible salmon populations become infectious, a fraction of infectious salmon die from ISA, viral dispersion and shedding occur; a fraction of each salmon class is removed (natural death and virus clearing); and susceptible salmon populations reproduce into the susceptible class. Typically, continuous-time differential equation models with similar well-defined distinct temporal phases are nonautonomous. Taking into account the temporal ordering of events in both farmed and wild salmon populations, we derive our ISA model with viral dispersal in the following three steps.

1. ISA transmission, ISA-induced death, viral shedding, and viral dispersion:

$$
\left.\begin{array}{ccc}
S_{(1)}^{F}= & S_{t}^{F}\left(\theta_{F} \varphi_{F}\left(I_{t}^{F}\right)+\left(1-\theta_{F}\right) \varphi_{V}^{F}\left(V_{t}^{F}\right)\right) \\
I_{(1)}^{F}= & S_{t}^{F}\left(\theta_{F} \widehat{\varphi}_{F}\left(I_{t}^{F}\right)+\left(1-\theta_{F}\right) \widehat{\varphi}_{V}^{F}\left(V_{t}^{F}\right)\right)+\widehat{\mu}_{F} I_{t}^{F} \\
V_{(1)}^{F}= & \left(1-m_{F}\right) V_{t}^{F}+\delta_{F} I_{t}^{F}+m_{W} V_{t}^{W}
\end{array}\right\}
$$

In the open net-cages, after ISA transmission, ISAinduced death, viral shedding, and viral dispersion, $S_{(1)}^{F}$ and $I_{(1)}^{F}$ denote the population sizes of susceptible and infectious farmed salmon, respectively, while $V_{(1)}^{F}$ denotes the population size of the ISA $v$.

2. Natural death (survival):

$$
\left.\begin{array}{rll}
S_{(2)}^{F} & =\widehat{d}_{F} S_{(1)}^{F} \\
I_{(2)}^{F} & =\widehat{d}_{F} I_{(1)}^{F} \\
V_{(2)}^{F} & =\widehat{d}_{V}^{F} V_{(1)}^{F}
\end{array}\right\}
$$

In the open net-cages, after ISA transmission, ISAinduced death, viral shedding, viral dispersion, and natural death, $S_{(2)}^{F}$ and $I_{(2)}^{F}$ denote the population sizes of susceptible and infectious farmed salmon, respectively, while $V_{(2)}^{F}$ denotes the population size of the ISAv.

3. Reproduction ( $S$ into $S)$ :

$$
\left.\begin{array}{ccc}
S_{(3)}^{F} & = & g_{F}\left(S_{t}^{F}\right)+S_{(2)}^{F} \\
I_{(3)}^{F} & = & I_{(2)}^{F} \\
V_{(3)}^{F} & = & V_{(2)}^{F}
\end{array}\right\}
$$

In the open net-cages, after ISA transmission, diseaseinduced death, viral shedding, viral dispersion, natural death, and reproduction, $S_{(3)}^{F}$ and $I_{(3)}^{F}$ denote the population sizes of susceptible and infectious farmed salmon, respectively, while $V_{(3)}^{F}$ denotes the population size of the ISA $v$.

The corresponding equations for the wild populations are similar. Our assumptions and notation lead to the following discrete-time farmed-wild salmon ISA model with viral dispersion:

$$
\begin{aligned}
S_{t+1}^{F}= & g_{F}\left(S_{t}^{F}\right)+\widehat{d}_{F} S_{t}^{F}\left(\theta_{F} \varphi_{F}\left(I_{t}^{F}\right)\right. \\
& \left.\quad+\left(1-\theta_{F}\right) \varphi_{V}^{F}\left(V_{t}^{F}\right)\right) \\
I_{t+1}^{F}= & \widehat{d}_{F}\left(S_{t}^{F}\left(\theta_{F} \widehat{\varphi}_{F}\left(I_{t}^{F}\right)+\left(1-\theta_{F}\right) \widehat{\varphi}_{V}^{F}\left(V_{t}^{F}\right)\right)\right. \\
& \left.+\widehat{\mu}_{F} I_{t}^{F}\right) \\
V_{t+1}^{F}= & \widehat{d}_{V}^{F}\left(\left(1-m_{F}\right) V_{t}^{F}+\delta_{F} I_{t}^{F}+m_{W} V_{t}^{W}\right) \\
S_{t+1}^{W}= & g_{W}\left(S_{t}^{W}\right)+\widehat{d}_{W} S_{t}^{W}\left(\theta_{W} \varphi_{W}\left(I_{t}^{W}\right)\right. \\
& \left.\quad+\left(1-\theta_{W}\right) \varphi_{V}^{W}\left(V_{t}^{W}\right)\right) \\
I_{t+1}^{W}= & \widehat{d}_{W}\left(S_{t}^{W}\left(\theta_{W} \widehat{\varphi}_{W}\left(I_{t}^{W}\right)+\left(1-\theta_{W}\right) \widehat{\varphi}_{V}^{W}\left(V_{t}^{W}\right)\right)\right. \\
& \left.\quad+\widehat{\mu}_{W} I_{t}^{W}\right) \\
V_{t+1}^{W}= & \widehat{d}_{V}^{W}\left(\left(1-m_{W}\right) V_{t}^{W}+\delta_{W} I_{t}^{W}+m_{F} V_{t}^{F}\right)
\end{aligned}
$$


where $t=0,1,2, \ldots$. We study Model (1) with initial conditions

$$
\left(S_{0}^{F}, I_{0}^{F}, V_{0}^{F}, S_{0}^{W}, I_{0}^{W}, V_{0}^{W}\right) \in \mathbb{R}_{+}^{6} .
$$

Model (1) predicts the vectors of population sizes in the farm, $\left(S_{t+1}^{F}, I_{t+1}^{F}, V_{t+1}^{F}\right)$, and nearby open water, $\left(S_{t+1}^{W}, I_{t+1}^{W}, V_{t+1}^{W}\right)$, at time $(t+1)$ from knowledge of the vectors of population sizes in the farm and open water, $\left(S_{t}^{F}, I_{t}^{F}, V_{t}^{F}\right)$ and $\left(S_{t}^{W}, I_{t}^{W}, V_{t}^{W}\right)$, at time $t$, where $t=$ $0,1,2, \ldots$. The unit of time depends on the specific salmon application. For simplicity, we take the unit of time in Model (1) to be a year. The basic reproduction number, $\mathcal{R}_{0}$, the lifetime production of ISA infections produced per ISA infectious salmon, is independent of the model's time scale. We will use Model (1) to compute $\mathcal{R}_{0}$. Model (1), a well-posed model, exhibits no unbounded salmon or virus population growth.

\section{Discrete-Time ISA Model without Dispersal}

The open net-cages and nearby open body of water of Model (1) are linked only by viral dispersion. When there are no dispersals and $m_{\Lambda}=0$ for each $\Lambda \in\{F, W\}$, the two subsystems decouple into two identical subsystems, except for the choice of parameters, and are given by the following single patch ISA $v$ model of the farmed (respectively, wild) salmon population in isolation when $\Lambda=F$ (respectively, when $\Lambda=W$ ):

$$
\begin{aligned}
S_{t+1}^{\Lambda}= & g_{\Lambda}\left(S_{t}^{\Lambda}\right)+\widehat{d}_{\Lambda} S_{t}^{\Lambda}\left(\theta_{\Lambda} \varphi_{\Lambda}\left(I_{t}^{\Lambda}\right)\right. \\
& \left.+\left(1-\theta_{\Lambda}\right) \varphi_{V}^{\Lambda}\left(V_{t}^{\Lambda}\right)\right) \\
I_{t+1}^{\Lambda}= & \widehat{d}_{\Lambda}\left(S_{t}^{\Lambda}\left(\theta_{\Lambda} \widehat{\varphi}_{\Lambda}\left(I_{t}^{\Lambda}\right)+\left(1-\theta_{\Lambda}\right) \hat{\varphi}_{V}^{\Lambda}\left(V_{t}^{\Lambda}\right)\right)\right. \\
& \left.+\widehat{\mu}_{\Lambda} I_{t}^{\Lambda}\right) \\
V_{t+1}^{\Lambda}= & \widehat{d}_{V}^{\Lambda}\left(V_{t}^{\Lambda}+\delta_{\Lambda} I_{t}^{\Lambda}\right),
\end{aligned}
$$

where $\Lambda \in\{F, W\}$. In [18], van den Driessche and Yakubu studied Model (2) with $\Lambda=F$.

Here, we consider the simplified demographic equation of Model (2) with no ISA disease $\left(I_{t}^{\Lambda}=V_{t}^{\Lambda}=0\right.$ for each $\Lambda \in\{F, W\})$, the following iteroparous Ricker equation:

$$
S_{t+1}^{\Lambda}=r_{\Lambda} S_{t}^{\Lambda} \exp \left(-b_{\Lambda} S_{t}^{\Lambda}\right)+\left(1-d_{\Lambda}\right) S_{t}^{\Lambda} .
$$

To illustrate salmon population cycles in Model (3) with $\Lambda=F$, in the farmed salmon population we choose the probability of natural death to be less than the probability of staying alive per unit time interval and set $d_{F}=0.4$. To scale the farmed salmon population sizes, we choose the scaling parameter, $b_{F}$, to equal 0.1. Figure 1 shows period-doubling bifurcations in Model (3) as the farmed salmon intrinsic growth rate, $r_{F}$, is varied between 20 and 470. Figure 1 shows windows of farmed salmon population cycles of period $1,2,4,8, \ldots$, and the range of the values of the parameter $r_{F}$ for the local asymptotic stability of the periodic farmed salmon population cycles. A period $k$ population cycle is a $k$-years population cycle, and Figure 1 shows simulations of the 2-years population cycle of pink salmon when $r_{F} \in(70,260)$ and the 4-years population cycle of the sockeye salmon when $r_{F} \in(270,370)$.

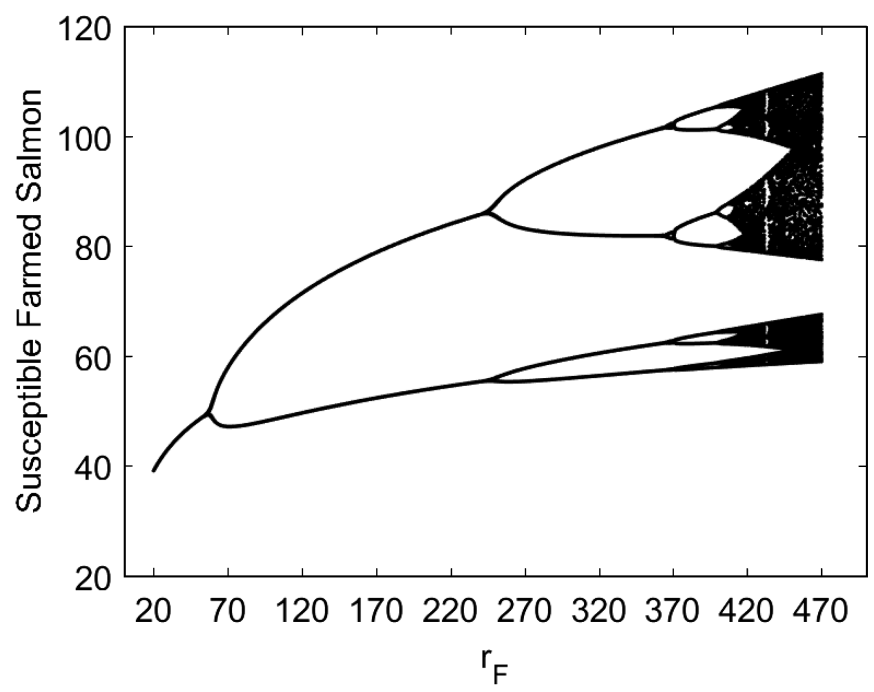

Figure 1. Period-doubling bifurcations in the farmed salmon of Model (3) with $\Lambda=F$, where $d_{F}=0.4$ and $b_{F}=0.1$, on the horizontal axis $20 \leq r_{F} \leq 470$ and on the vertical axis $20 \leq S_{F} \leq 120$.

The basic reproduction number $\mathcal{R}_{0}^{\Lambda, 3}$. Assuming ISA $v$ shedding is not a new infection [4], [5], in [18], van den Driessche and Yakubu used the next generation matrix method to compute the basic reproduction number, $\mathcal{R}_{0}^{\Lambda, 3}$, for the three-dimensional Model (2) for $\Lambda=F$ or $\Lambda=W$, where the salmon demographic threshold $\mathcal{R}_{d}^{\Lambda}>1$ and equation (3) has a unique positive locally asymptotically stable period $k$ salmon population cycle. Thus, when the farmed (respectively, wild) salmon population is in isolation and $\mathcal{R}_{d}^{\Lambda}>1$, then the ISA disease-free period $k$ isolated farmed (respectively, wild) salmon population cycle of Model (2) is locally asymptotically stable, and the ISA $v$ disease epidemic is eliminated in the population when $\mathcal{R}_{0}^{\Lambda, 3}<1$, where $\Lambda=F$ (respectively, $\Lambda=W$ ). However, the ISA disease-free period $k$ isolated farmed (respectively, wild) salmon population cycle is unstable and ISA invades the isolated population of the three-dimensional Model (2) when $\mathcal{R}_{0}^{\Lambda, 3}>1$.

Illustrative examples in single patch model without dispersion. To illustrate ISA elimination and endemicity in Model (2), we assume that the ISA infections are modeled as Poisson processes and let

$$
\begin{aligned}
\Lambda=F, \quad \varphi_{\Lambda}\left(I^{\Lambda}\right)=\exp \left(-\beta_{\Lambda} I^{\Lambda}\right), \\
\text { and } \varphi_{V}^{\Lambda}\left(V^{\Lambda}\right)=\exp \left(-\beta_{V}^{\Lambda} V^{\Lambda}\right)
\end{aligned}
$$

where

$$
b_{F}=0.1, \quad d_{F}=\delta_{F}=0.4, \quad d_{V}^{F}=0.2, \quad r_{F}=72,
$$




$$
\beta_{F}=0.01, \quad \beta_{V}^{F}=0.01, \quad \widehat{\mu}_{F}=0.3, \quad \text { and } \quad \theta_{F}=0.6 \text {. }
$$

With our choice of parameters, $\mathcal{R}_{d}^{F}=\frac{r_{F}}{d_{F}}=180>e^{\frac{2}{d_{F}}}=$ $e^{5} \approx 148.4$, and Model (3), the ISA disease-free equation has a unique positive asymptotically stable period 2 farmed salmon population cycle (see Figures 1 and 2). Figure 2 shows that $\mathcal{R}_{0}^{F, 3}<1$ (ISA is eliminated), and the ISA disease-free period 2 farmed population cycle is asymptotically stable.
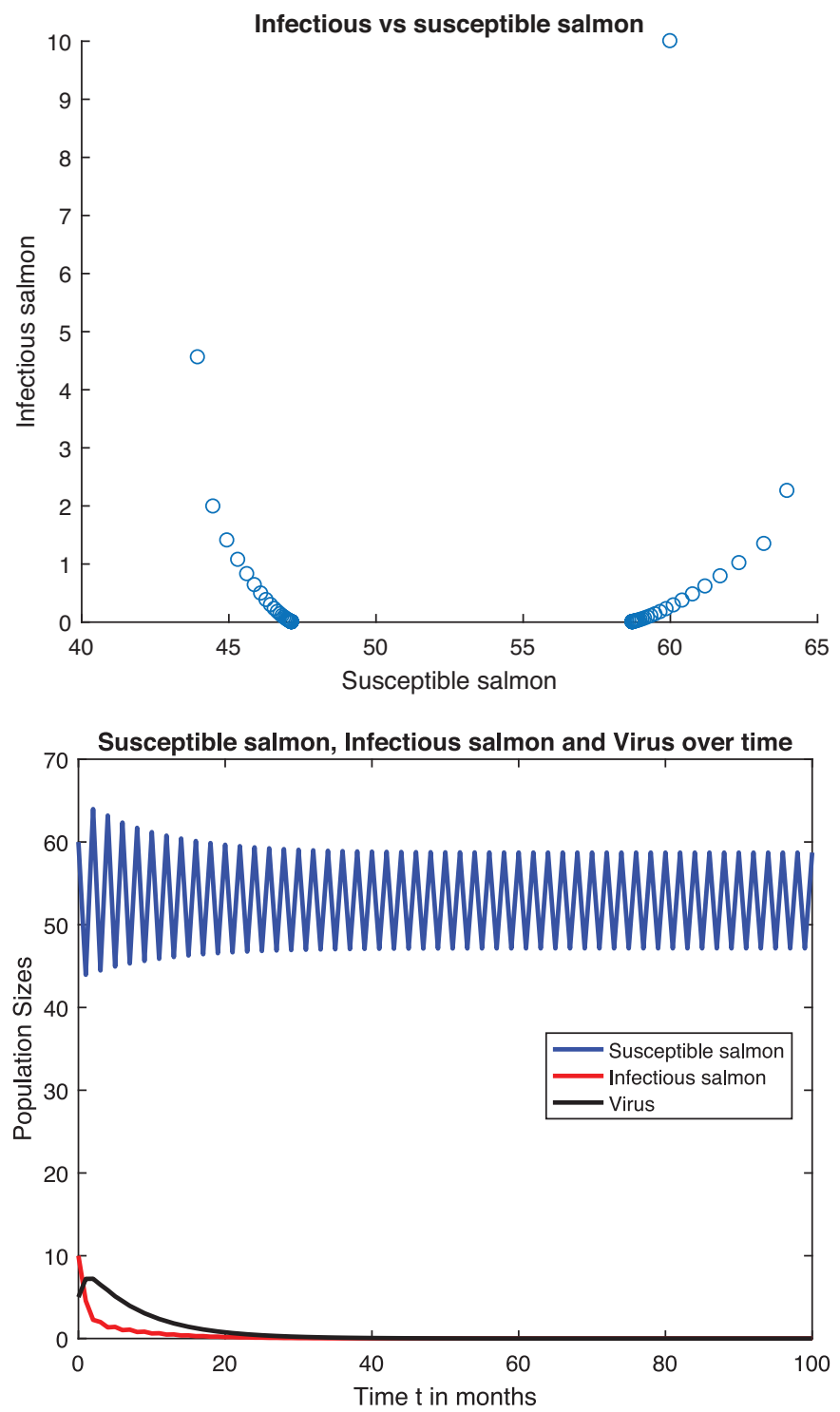

Figure 2. $\mathcal{R}_{0}^{F, 3}<1$ and Model (2) has a locally asymptotically stable disease-free period 2 farmed salmon population cycle, where

$$
\begin{gathered}
b_{F}=0.1, d_{F}=\delta_{F}=0.4, d_{V}^{F}=0.2 \\
r_{F}=72, \beta_{F}=0.01, \beta_{V}^{F}=0.01, \text { and } \theta_{F}=0.6 .
\end{gathered}
$$

To illustrate the impact of higher values of the ISA $v$ infection rate, $\beta_{F}$, on Figure 2, we increase $\beta_{F}$ to $\beta_{F}=0.04$ and keep all the other parameters fixed at their current values in Figure 2. In this case, Figure 3 shows that $\mathcal{R}_{0}^{F, 3}>1$ (ISA disease is endemic), the ISA disease-free period 2 farmed population cycle is unstable, and Model (2) has an ISA endemic period 4 population cycle. It is interesting to note that, in this example, the 2-years period of the demographic farmed salmon population cycle is not equal to the 4-years period of the ISA endemic disease dynamics. How do ISA infections influence the 4-years cycle of sockeye salmon?
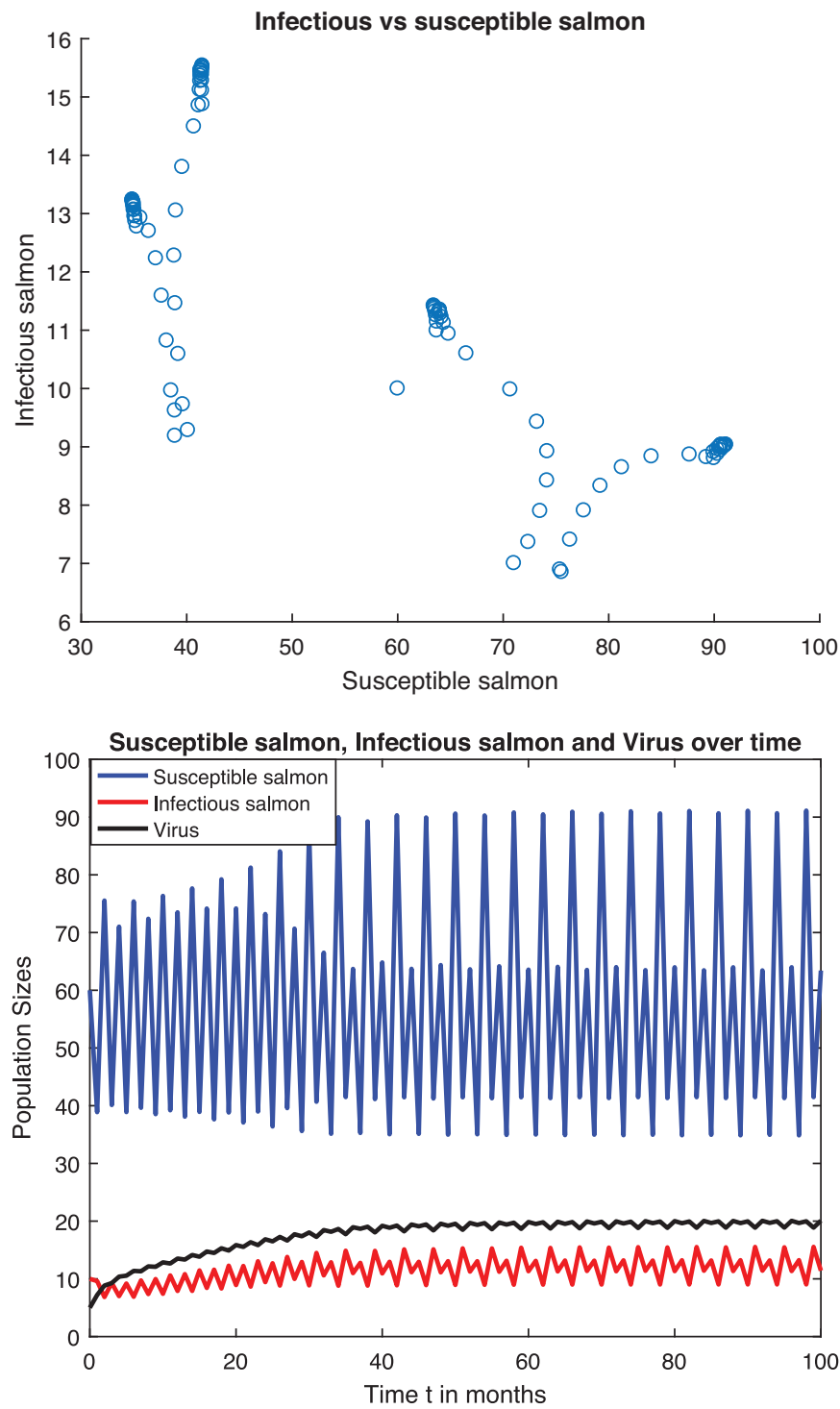

Figure 3. $\mathcal{R}_{0}^{F, 3}>1$ and Model (2) has an asymptotically stable endemic period 4 population cycle, where $\beta_{F}=0.04$ and all the other parameters are kept fixed at their current values in Figure 2. 


\section{ISA Model with Only Farmed Salmon and Viral Dispersal}

When the farmed salmon population is present in the open net-cages, and the wild salmon population is missing in the nearby open body of water, then $S_{t}^{W}=I_{t}^{W}=0$, and Model (1) reduces to the following four-dimensional ISA model with only farmed salmon that are confined to the open net-cages, and with dispersion of ISA $v$ between the open net-cages and the nearby open body of water:

$$
\begin{aligned}
S_{t+1}^{F}= & g_{F}\left(S_{t}^{F}\right)+\widehat{d}_{F} S_{t}^{F}\left(\theta_{F} \varphi_{F}\left(I_{t}^{F}\right)\right. \\
& \left.\quad+\left(1-\theta_{F}\right) \varphi_{V}^{F}\left(V_{t}^{F}\right)\right) \\
I_{t+1}^{F}= & \widehat{d}_{F}\left(S_{t}^{F}\left(\theta_{F} \widehat{\varphi}_{F}\left(I_{t}^{F}\right)+\left(1-\theta_{F}\right) \hat{\varphi}_{V}^{F}\left(V_{t}^{F}\right)\right)\right. \\
& \left.+\widehat{\mu}_{F} I_{t}^{F}\right) \\
V_{t+1}^{F}= & \widehat{d}_{V}^{F}\left(\left(1-m_{F}\right) V_{t}^{F}+\delta_{F} I_{t}^{F}+m_{W} V_{t}^{W}\right) \\
V_{t+1}^{W}= & \widehat{d}_{V}^{W}\left(\left(1-m_{W}\right) V_{t}^{W}+m_{F} V_{t}^{F}\right)
\end{aligned}
$$

In Model (4), the shedding of ISA $v$ by the infected farm salmon makes the open net-cages a suitable environment for the reproduction of the ISA $v$. However, the absence of wild salmon makes the nearby open water an unsuitable barren environment that is devoid of resources for the reproduction of the ISA $v$. What is the relationship between the basic reproduction number of Model (4), $\mathcal{R}_{0}^{F, 4}$, and that of Model (3), $\mathcal{R}_{0}^{F, 3}$ ? In the absence of wild salmon in close proximity to an ISA-infected salmon farm, can ISA $v$ dispersion eliminate the ISA from the farm? To answer this question, we first note that Models (2) and (4) share the same ISA disease-free $\left(I_{t}^{F}=V_{t}^{F}=V_{t}^{W}=0\right)$ demographic equation, Model (3). Next, we compute the ISA diseasefree equilibrium points.

$D F E$ for ISA model with only farmed salmon and viral dispersal. Model (4) has only the trivial ISA disease-free equilibrium (DFE),

$$
\left(S_{-}^{F}, I_{-}^{F}, V_{-}^{F}, V_{-}^{W}\right)=(0,0,0,0)
$$

when $\mathcal{R}_{d}^{F}<1$. As in Model (2), Model (4) has two ISA DFEs, the trivial ISA DFE and

$$
\left(S_{-}^{F}, I_{-}^{F}, V_{-}^{F}, V_{-}^{W}\right)=\left(\frac{\ln \mathcal{R}_{d}^{F}}{b_{F}}, 0,0,0\right),
$$

when $1<\mathcal{R}_{d}^{F}$. However, when $\mathcal{R}_{d}^{F}>e^{\frac{2}{d_{F}}}$ it is possible for Model (4) to have an asymptotically positive period $k>1$ population cycle ISA DFE.

To study Model (4) with persistent fixed or cyclic farmed salmon populations, we assume that $\mathcal{R}_{d}^{F}>1$ and the ISA disease-free equation has a unique positive asymptotically stable period $k$ farmed salmon population cycle,

$$
\left\{\bar{s}_{1}^{F}, \bar{s}_{2}, \ldots, \bar{s}_{k}^{F}\right\},
$$

where $k \in\{1,2, \ldots\}$. In the next section, we compute $\mathcal{R}_{0}^{F, 4}$, the basic reproduction number for the four-dimensional Model (4) with only farmed salmon and viral dispersion.
The basic reproduction number $\mathcal{R}_{0}^{F, 4}$. Proceeding as in [18] we obtain $\mathcal{R}_{0}^{F, 4}$, the basic reproduction number for the four-dimensional Model (4) with only farmed salmon and viral dispersion. That is, when $\mathcal{R}_{d}^{F}>1$ and the salmon population is present only in the open net-cages, then the ISA disease-free period $k$ farmed salmon population cycle of Model (4),

$$
\left\{\left(\bar{s}_{1}^{F}, 0,0,0\right),\left(\bar{s}_{2}^{F}, 0,0,0\right), \ldots,\left(\bar{s}_{k}^{F}, 0,0,0\right)\right\},
$$

is locally asymptotically stable, and the ISA epidemic is eliminated in the farmed salmon population when $\mathcal{R}_{0}^{F, 4}<$ 1 , where there is viral dispersion. However,

$$
\left\{\left(\bar{s}_{1}^{F}, 0,0,0\right),\left(\bar{s}_{2}^{F}, 0,0,0\right), \ldots,\left(\bar{s}_{k}^{F}, 0,0,0\right)\right\}
$$

is unstable, and the ISA disease persists in the farmed salmon population of the four-dimensional Model (2) when $\mathcal{R}_{0}^{F, 4}>1$.

When $1<\mathcal{R}_{d}^{F}<e^{\frac{2}{d_{F}}}$, assuming that virus shedding is not a new infection, and proceeding exactly as in the case with no viral dispersion, we obtain that

$$
\mathcal{R}_{0}^{F, 4}>\mathcal{R}_{0}^{F, 3}=\mathcal{R}_{0 I}^{F, 3}+\mathcal{R}_{0 V}^{F, 3} .
$$

That is, unlike in the ODE model of [12], in Model (4), $\mathcal{R}_{0}^{F, 4}>1$ whenever $\mathcal{R}_{0}^{F, 3}>1$. Thus, in the absence of the wild salmon, linear dispersion of the virus cannot eliminate ISA from an infected farm. However, in agreement with [12], we obtain that $\mathcal{R}_{0}^{F, 4}<1$ implies $\mathcal{R}_{0}^{F, 3}<1$. That is, in the absence of wild salmon, the susceptible farmed salmon population in the open net-cages is not a rich enough resource to overcome the deleterious effects of being coupled to the barren nearby open body of water. Mathematically, it is possible to have $\mathcal{R}_{0}^{F, 4}>1$ while $\mathcal{R}_{0}^{F, 3}<1$.

$\mathcal{R}_{0}^{F, 4}$ versus $\mathcal{R}_{0}^{F, 3}$. Can viral dispersion stabilize the endemic period 4 population cycle of Figure 3 ? To study the impact of viral dispersion on Figure 3, in Model (4) we let

$$
d_{V}^{W}=0.4, \quad m_{F}=0.6>m_{W}=0.001
$$

and keep all the other parameters fixed at their current values in Figure 3. In this case, the proportion of ISA $v$ that disperses from the farmed cages far exceeds the proportion that disperses from the open water to the cages, and Figure 4 shows that the virus clearing in the nearby open water helps stabilize the 4-years ISA endemic cycle of Figure 3 to a 2-years ISA endemic cycle. That is, higher proportion of viral dispersion from the farmed cages is capable of stabilizing ISA $v$ disease dynamics via what appears to be a period-doubling bifurcation reversal. To explore the effect of higher proportional virus dispersion from the open water to the ISA infected farm of Figure 3, we let

$$
m_{F}=0.001<m_{W}=0.6
$$



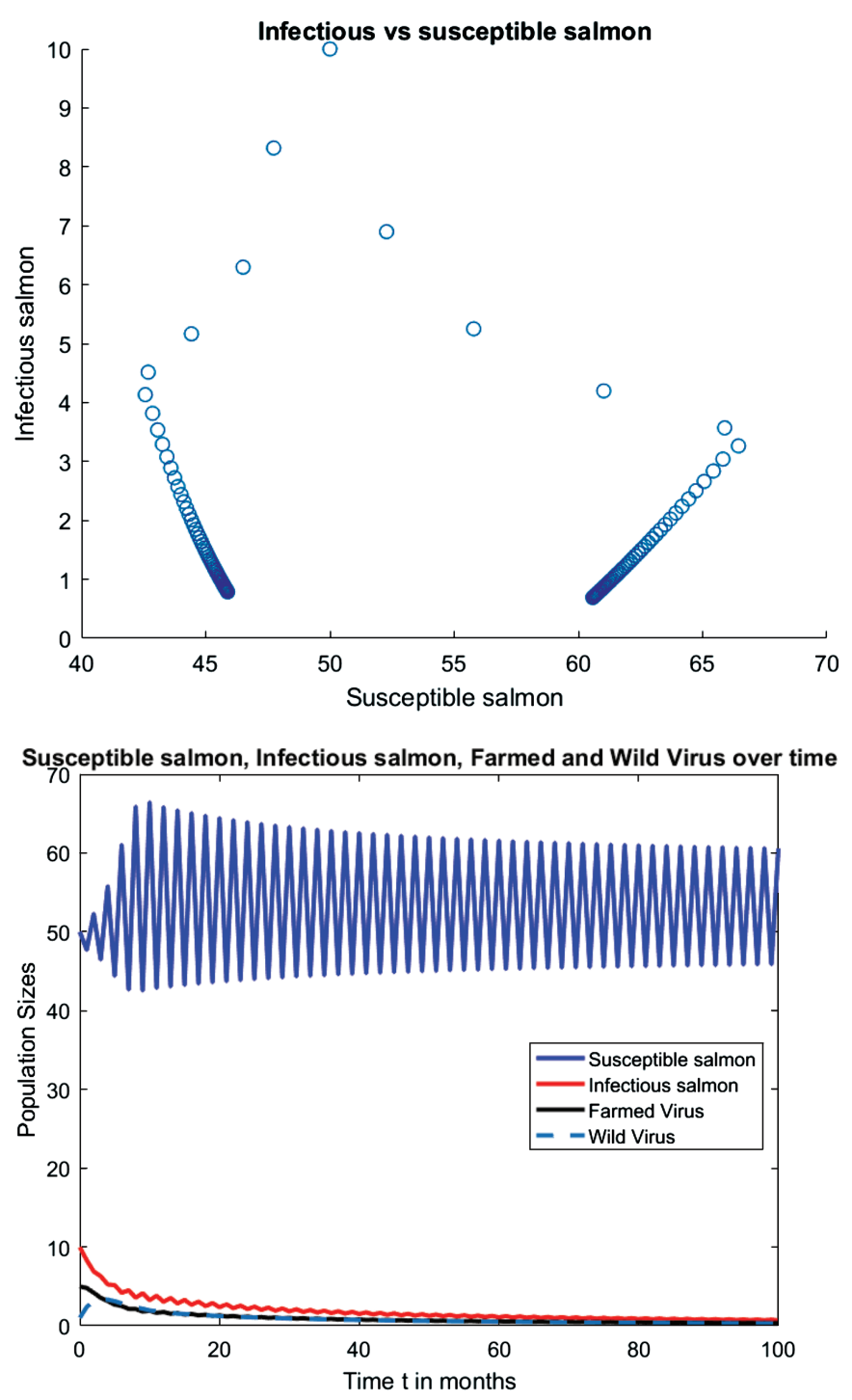

Figure 4. $\mathcal{R}_{0}^{F, 4}>1$ and Model (4) has an asymptotically stable endemic period 2 population cycle, where

$$
d_{V}^{W}=0.4, m_{F}=0.6, m_{W}=0.001
$$

and all the other parameters are kept fixed at their current values in Figure 3.

and keep all the other parameters fixed at their current values in Figure 3. In this case, the proportion of ISA $v$ that disperses from the farmed cages is much lower than the proportion that disperses from the open water to the cages, and Figure 5 shows that lower proportion of virus dispersion from the open water preserves the 4-years ISA endemic cycle of Figure 3.

\section{$D F E$ for ISAv Farmed-Wild Salmon Viral Dispersal Model}

Model (1) has only the trivial ISA disease-free equilibrium (DFE),

$$
\Sigma_{00}=\left(S_{-}^{F}, I_{-}^{F}, V_{-}^{F}, S_{-}^{W}, I_{-}^{W}, V_{-}^{W}\right)=(0,0,0,0,0,0)
$$
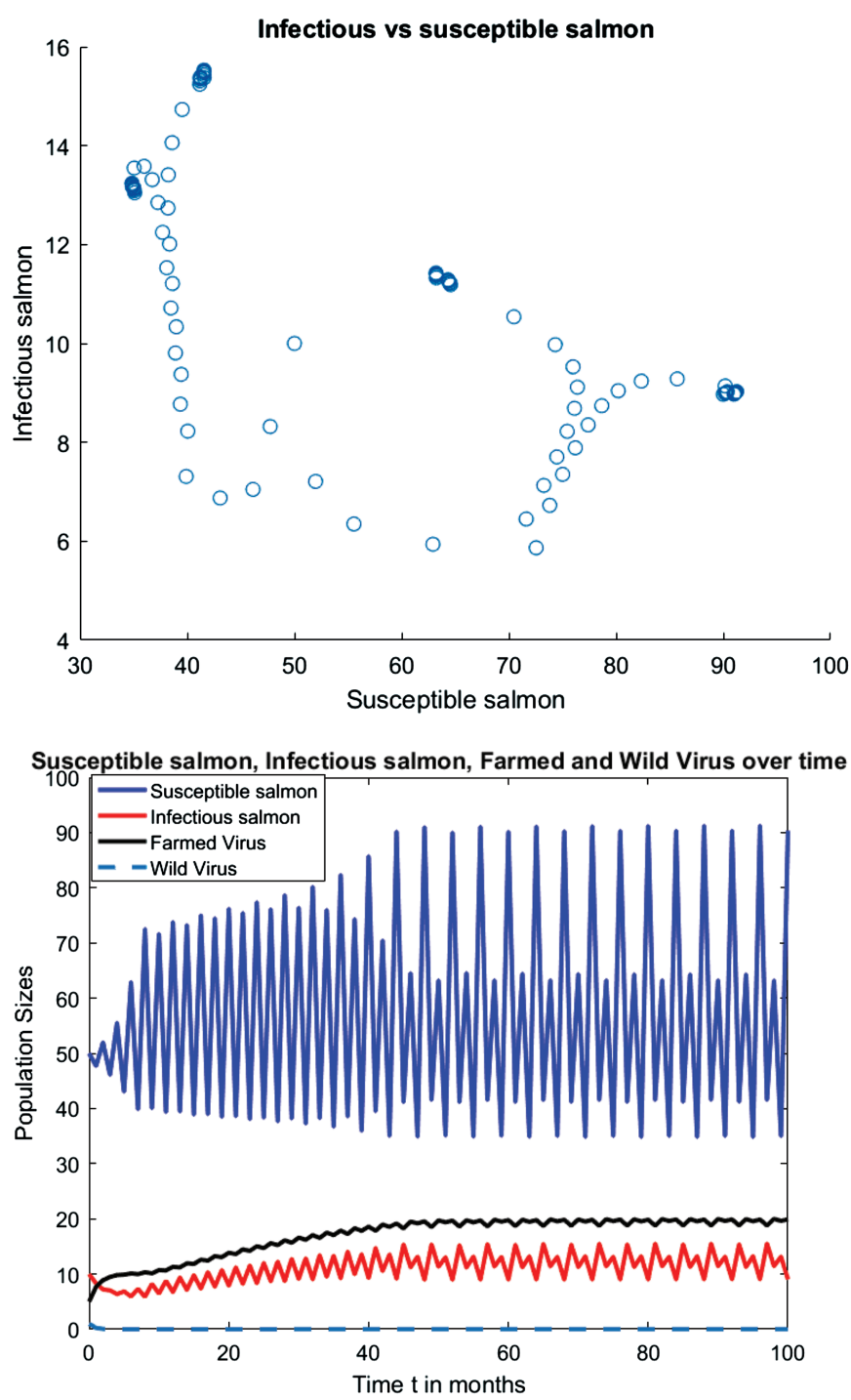

Figure 5. $\mathcal{R}_{0}^{F, 4}>1$ and Model (4) has an asymptotically stable endemic period 4 population cycle, where

$$
d_{V}^{W}=0.4, m_{F}=0.001, m_{W}=0.6
$$

and all the other parameters are kept fixed at their current values in Figure 3.

when $\mathcal{R}_{d}^{F}<1$ and $\mathcal{R}_{d}^{W}<1$. Recall that $\mathcal{R}_{d}^{F}<$ 1 (respectively, $\mathcal{R}_{d}^{W}<1$ ) implies the extinction of the farmed (respectively, wild) salmon population in the disease-free equation. Hence, $\Sigma_{00}$ is globally asymptotically stable, and the farmed-wild salmon system collapses in Model (1) when $\mathcal{R}_{d}^{F}<1$ and $\mathcal{R}_{d}^{W}<1$.

When $\mathcal{R}_{d}^{F}>1$ and $\mathcal{R}_{d}^{W}<1$, then the wild salmon population goes extinct in the nearby water while the farmed salmon population persists in the open cages. Similarly, when $\mathcal{R}_{d}^{F}<1$ and $\mathcal{R}_{d}^{W}>1$, then the farmed salmon population goes extinct in the open cages while the wild salmon population persists in the nearby water. Consequently, Model (4) is the "limiting" system of Model (1) 
whenever either $\mathcal{R}_{d}^{F}>1$ and $\mathcal{R}_{d}^{W}<1$ or $\mathcal{R}_{d}^{F}<1$ and $\mathcal{R}_{d}^{W}>1$. Thus, when $\mathcal{R}_{d}^{F}>1$ and $\mathcal{R}_{d}^{W}<1$, in addition to $\Sigma_{00}$,

$$
\Sigma_{F 0}=\left(S_{-}^{F}, I_{-}^{F}, V_{-}^{F}, S_{-}^{W}, I_{-}^{W}, V_{-}^{W}\right)=\left(\frac{\ln \mathcal{R}_{d}^{F}}{b_{F}}, 0,0,0,0,0\right)
$$

is an ISA DFE of Model (1) with susceptible farmed salmon. Similarly, when $\mathcal{R}_{d}^{F}<1$ and $\mathcal{R}_{d}^{W}>1$, in addition to $\Sigma_{00}$,

$$
\Sigma_{0 W}=\left(S_{-}^{F}, I_{-}^{F}, V_{-}^{F}, S_{-}^{W}, I_{-}^{W}, V_{-}^{W}\right)=\left(0,0,0, \frac{\ln \mathcal{R}_{d}^{W}}{b_{W}}, 0,0\right)
$$

is an ISA DFE of Model (1) with susceptible wild salmon. In either case, $\Sigma_{00}$ is unstable. When the salmon population persists on a fixed point or a period $k$ population cycle attractor in the disease-free equation, $\mathcal{R}_{0}^{F, 4}$ determines the stability of $\Sigma_{F 0}$ while $\mathcal{R}_{0}^{W, 4}$ determines the stability of $\Sigma_{0 W}$.

When $\mathcal{R}_{d}^{F}>1$ and $\mathcal{R}_{d}^{W}>1$, in addition to the unstable trivial ISA DFE, $\Sigma_{00}$, Model (1) has the ISA DFE with susceptible farmed and wild salmon populations,

$$
\begin{aligned}
\Sigma_{F W}=\left(S_{-}^{F}, I_{-}^{F}, V_{-}^{F}, S_{-}^{W},\right. & \left.I_{-}^{W}, V_{-}^{W}\right) \\
& =\left(\frac{\ln \mathcal{R}_{d}^{F}}{b_{F}}, 0,0, \frac{\ln \mathcal{R}_{d}^{W}}{b_{W}}, 0,0\right) .
\end{aligned}
$$

To study Model (1) with persistent fixed or periodic farmed and wild salmon populations, we assume that $\mathcal{R}_{d}^{F}>1$ and $\mathcal{R}_{d}^{W}>1$, and the disease-free system of two equations has a unique positive asymptotically stable period $k$ farmed and wild salmon population cycle,

$$
\left\{\left(\bar{s}_{1}^{F}, \bar{s}_{1}^{W}\right),\left(\bar{s}_{2}^{F}, \bar{s}_{2}^{W}\right), \ldots,\left(\bar{s}_{k}^{F}, \bar{s}_{k}^{W}\right)\right\}
$$

where $k \in\{1,2, \ldots\}$. In the next section, we compute $\mathcal{R}_{0}^{6}$, the basic reproduction number for the full six-dimensional farmed and wild salmon Model (1) with dispersion.

The basic reproduction number $\mathcal{R}_{0}^{6}$. Proceeding as in the previous sections we obtain $\mathcal{R}_{0}^{6}$, the basic reproduction number for the six-dimensional Model (1) with both farmed and wild salmon populations and viral dispersion. When $\mathcal{R}_{d}^{F}>1, \mathcal{R}_{d}^{W}>1$, and the salmon populations persist in the open net-cages and the nearby open water, the ISA disease-free period $k$ farmed and wild salmon population cycle of Model (1),

$$
\begin{array}{r}
\left\{\left(\bar{s}_{1}^{F}, 0,0, \bar{s}_{1}^{W}, 0,0\right),\left(\bar{s}_{2}^{F}, 0,0, \bar{s}_{2}^{W}, 0,0\right),\right. \\
\left.\ldots,\left(\bar{s}_{k}^{F}, 0,0, \bar{s}_{k}^{W}, 0,0\right)\right\},
\end{array}
$$

is locally asymptotically stable, and the ISA epidemic is eradicated in both farmed and wild salmon populations when $\mathcal{R}_{0}^{6}<1$. However,

$$
\begin{aligned}
\left\{\left(\bar{s}_{1}^{F}, 0,0, \bar{s}_{1}^{W}, 0,0\right),\left(\bar{s}_{2}^{F}, 0,0, \bar{s}_{2}^{W}, 0,0\right),\right. \\
\left.\ldots,\left(\bar{s}_{k}^{F}, 0,0, \bar{s}_{k}^{W}, 0,0\right)\right\}
\end{aligned}
$$

is unstable and the disease invades the salmon populations when $\mathcal{R}_{0}^{6}>1$. In the next section, we study the impact of the presence of the wild salmon population in the nearby body of water on the endemic period 2 ISA population cycle of Figure 4.

Illustrative examples in farmed-wild salmon model with dispersion. To illustrate the impact of viral dispersion in the presence of wild salmon in close proximity to the ISAinfected salmon farm of Figure 4, in Model (1), we assume that for each $\Lambda \in\{F, W\}$, ISA infections are modeled as Poisson processes, and let

$$
\begin{gathered}
\beta_{W}=\beta_{V}^{W}=0.01, \quad d_{V}^{W}=0.7, \\
\delta_{W}=0.01, \quad b_{W}=0.1, \quad d_{W}=0.8, \\
r_{W}=60, \quad \mu_{W}=0.1, \quad \theta_{W}=0.01, \\
\text { and } m_{F}=0.6>m_{W}=0.001,
\end{gathered}
$$

where all the other model parameters are kept fixed at their current values in Figure 4. With our choice of parameters, $\mathcal{R}_{d}^{F}>e^{\frac{2}{d_{F}}}, \mathcal{R}_{d}^{W}=\frac{r_{W}}{d_{W}}>e^{\frac{2}{d_{W}}}$, and the ISA disease-free equation for the farmed salmon population is a unique positive asymptotically stable period 2 population cycle (see Figure 1), while that of the wild salmon is a unique positive asymptotically stable period 8 population cycle (not shown).

ISA has caused high death rates in marine farmed Atlantic salmon, but the virus has not been associated with die-offs in wild salmon populations. We capture this with our choice of parameters, ISA $v$ is enzootic in the farmed salmon, $\mathcal{R}_{0}^{F, 3}>1$, and Figure 4 shows that the predispersion dynamics of the isolated farmed salmon is an ISA endemic period 2 population cycle. However, without viral dispersion, ISA $v$ is cleared in the isolated wild salmon population and $\mathcal{R}_{0}^{W, 3}<1$ (not shown). When the proportion of ISA $v$ that disperses from the ISA enzootic farmed cages far exceeds the proportion that disperses from the open water to the cages $\left(m_{F}=0.6>m_{W}=0.001\right)$, Figure 6 shows that it is possible for the viral-linked full system to change from an ISA-free wild salmon population without viral dispersion to an ISA-infected wild salmon population with dispersion, where $\mathcal{R}_{0}^{6}>1$. However, when we set $m_{F}=0.001<m_{W}=0.6$ and keep all the other parameters fixed at their current values in Figure 6, then the proportion of ISA $v$ that disperses from the farmed cages is much lower than the proportion that disperses from the open water to the cages. In this case, dispersion does not alter the predispersion source-sink dynamics (not shown). That is, 
in the event of an ISA outbreak in a farmed salmon population, a higher proportion of ISA viral dispersion from the infected farm could put the nearby wild salmon population at risk of the ISA disease.
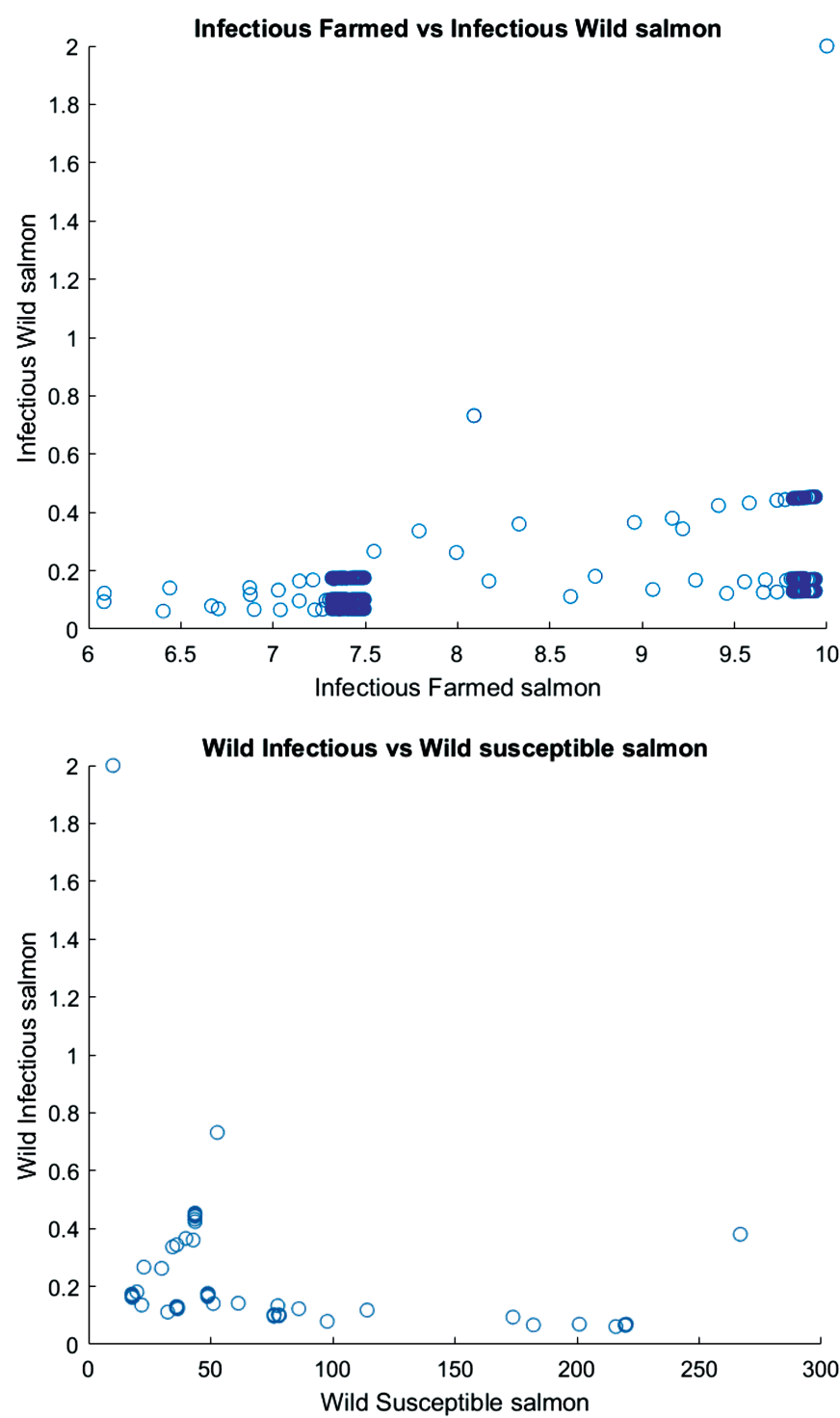

Figure 6. $\mathcal{R}_{0}^{6}>1$ and Model (1) has an asymptotically stable ISA endemic period 6 in both farmed and wild salmon populations, where $\beta_{W}=\beta_{V}^{W}=0.01, d_{V}^{W}=0.7, \delta_{W}=0.01$, $b_{W}=0.1, d_{W}=0.8, r_{W}=60, \mu_{W}=0.1, \theta_{W}=0.01$, $m_{F}=0.6>m_{W}=0.001$, and all the other model parameters are kept fixed at their current values in Figure 4.

\section{Concluding Remarks}

Salmon populations are known to exhibit patterns of cyclic dominance of subpopulations. To study the impact of ISA viral disease and the patterns of cyclic dynamics in salmon populations, we introduce a viral dispersal-linked farmed-wild salmon ISA discrete-time infectious disease model with intrinsically generated demographic population cycles (cyclic disease-free dynamics). Unlike the logistic growth dynamics in the ODE model of [12], the susceptible farmed and wild salmon populations in our model exhibit cyclic dynamics via the Ricker growth or recruitment (or birth) functions. Furthermore, in our ISA model, the proportion of ISA virus that disperses from the farmed cages to the open water is not equal to the proportion that disperses from the open water to the cages.

We compute the basic reproduction number, $\mathcal{R}_{0}$, for ISA disease elimination or invasion. When $\mathcal{R}_{0}<1$ and the Ricker demographic equation has a locally asymptotically stable $k$-years population cycle, we prove the local asymptotic stability of the dispersal-linked farmed-wild salmon ISA disease-free $k$-years cycle (with and without the wild salmon present). That is, the ISA is eliminated in the farmed and wild salmon populations whenever $\mathcal{R}_{0}<1$. Also, under the same $k$-years Ricker demographic assumption, we prove that the ISA disease-free $k$-years population cycle is unstable and the ISA invades the farmed and wild salmon populations when $\mathcal{R}_{0}>1$.

In the absence of wild salmon in close proximity to farmed salmon cages, we illustrate in Figures 3 and 4 that higher viral dispersion from ISA-infected farmed cages is capable of stabilizing ISA $v$ cyclic disease dynamics via what appears to be a period-doubling bifurcation reversal, while lower viral dispersion from the cages preserves the predispersion ISA enzootic cyclic dynamics (see Figure 5). Furthermore, in Figure 6, we illustrate that higher proportion of ISA viral dispersion from an ISA-infected salmon farm could put the nearby wild salmon population at risk of the ISA disease. Whenever there is an ISA disease outbreak on a salmon farm and data for estimating the model parameters is available, our computed $\mathcal{R}_{0}$ can be used to determine when intervention measures will have the ISA disease outbreak under control. Theoretical results on the relationship between the period of the Ricker demographic ISA disease-free period $k$ salmon population cycles and the period of the endemic cycles of the viral dispersallinked farmed-wild salmon ISA disease models are open.

ACKNOWLEDGMENT. The author was partially supported by NSF Computational Sustainability Grant CCF-1522054 and NSF Award DMS-1743144. He thanks the reviewers for the useful suggestions.

\section{References}

[1] Allen L, van den Driessche P. The basic reproduction number in some discrete-time epidemic models, J. Diff. Eqns. \& Appl., 14(10-11):1127-1147, 2008 MR2447189

[2] Brauer F, Feng Z, Castillo-Chavez C. Discrete epidemic models, Math. Biosc. Eng., (7):1-15, 2010. MR2654277 
[3] Castillo-Chavez C, Yakubu A-A. Dispersal, disease and life-history evolution, Math. Biosc., (173):35-53, 2001. MR1860421

[4] Cushing JM, Diekmann O. The many guises of $\mathcal{R}_{0}$ (a diadactic note), J. Theor. Biol., (404):295-302, 2016. MR3521414

[5] Diekmann O, Heesterbeek JAP, Metz JAJ. On the definition and computation of the basic reproduction ratio $\mathcal{R}_{0}$ in models for infectious diseases in heterogeneous populations, J. Math. Biol., (28):365-382, 1990. MR1057044

[6] Falk K, Namork E, Rimstad E, Mjaaland S, Dannevig BH. Characterization of Infectious Salmon Anemia Virus [1997], an Orthomyxo-Like Virus Isolated from Atlantic Salmon (Salmo salar L.), Journal of Virology, 71(12):90169023.

[7] FAO. Cultured Aquatic Species Information Programme. http://www. fao.org/fishery/culturedspecies /Salmo_salar/en\#tcNA00D6

[8] Kibenge FSB, Garate ON, Johnson GR, Arriagada R, Kibenge MJT, Wadowska D. Isolation and identification of infectious salmon anaemia virus (ISA $v$ ) from Coho salmon in Chile, Diseases of Aquatic Organisms, 45(1):9-18, 2001.

[9] Krkosek M, Lewis MA, Volpe JP. Transmission dynamics of parasitic sea lice from farm to wild salmon, Proc. R. Soc. B., (272):689-696, 2005.

[10] Levy DA, Wood CC. Review of proposed mechanisms for sockeye salmon population cycles in the Fraser river, Bull. Math. Biol., 54(2-3):241-261, 1992.

[11] May RM. Simple mathematical models with very complicated dynamics, Nature, (261):459-467, 1976.

[12] Milliken E, Pilyugin SS. A model of infectious salmon anemia virus with viral diffusion between wild and farmed patches, Discrete \& Cont. Dyn. Sys. B, 21(6):1869-1893, 2016. MR3543613

[13] Mowi, Salmon Farming Industry Handbook 2019. https://corpsite.azureedge.net/corpsite/wp -content/uploads/2019/06/Sa Imon-Industry -Handbook-2019.pdf

[14] Myers RA, Mertz G, Bridson JM, Bradford MJ. Simple dynamics underlie sockeye salmon (Oncorhynchus nerka) cycles, Canadian Journal of Fisheries and Aquatic Sciences, (55):2355-2364, 1998.

[15] Ricker WE. Stock and recruitment, J. Fish Res. Board, Can., (11):559-623, 1954.

[16] OurWorldInData. Meat and Seafood Production and Consumption. https://ourworldindata.org/meat -and-seafood-production-consumption

[17] van den Driessche P, Yakubu A-A. Disease extinction versus persistence in discrete-time epidemic models, Bull. Math. Biol., 81(11):4412-4446, 2019. MR4034832

[18] van den Driessche P, Yakubu A-A. Demographic Population Cycles and $R_{0}$ in Discrete-Time Epidemic Models, $J$. Biol. Dyn., (13):179-200, 2019. PMID: 30373469

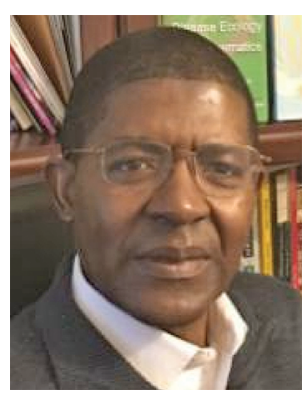

Abdul-Aziz Yakubu

Credits

Opener image is courtesy of Getty.

All other figures and photos are courtesy of the author.

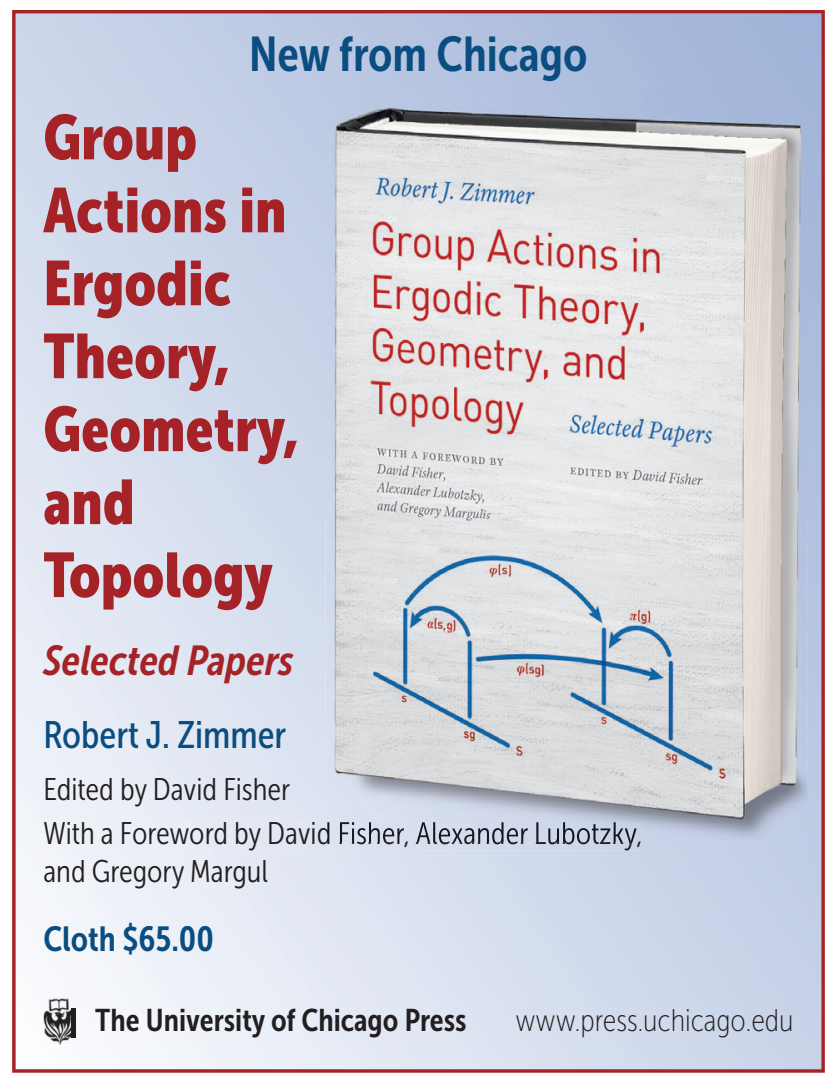

